\title{
A multipartite version of the Hajnal-Szemerédi theorem for graphs and hypergraphs *
}

\author{
Allan Lo ${ }^{\dagger}$ \\ School of Mathematics, University of Birmingham, \\ Birmingham, B15 2TT, UK \\ s.a.lo@bham.ac.uk \\ Klas Markström \\ Department of Mathematics and Mathematical Statistics, \\ Umeå University, S-901 87 Umeå, Sweden \\ klas.markstrom@math.umu.se
}

October 6, 2018

\begin{abstract}
A perfect $K_{t}$-matching in a graph $G$ is a spanning subgraph consisting of vertex disjoint copies of $K_{t}$. A classic theorem of Hajnal and Szemerédi states that if $G$ is a graph of order $n$ with minimum degree $\delta(G) \geq(t-$ 1) $n / t$ and $t \mid n$, then $G$ contains a perfect $K_{t}$-matching. Let $G$ be a $t$-partite graph with vertex classes $V_{1}, \ldots, V_{t}$ each of size $n$. We show that for any $\gamma>0$ if every vertex $x \in V_{i}$ is joined to at least $((t-1) / t+\gamma) n$ vertices of $V_{j}$ for each $j \neq i$, then $G$ contains a perfect $K_{t}$-matching, provided $n$ is large enough. Thus, we verify a conjecture of Fisher [6] asymptotically. Furthermore, we consider a generalisation to hypergraphs in terms of the codegree.
\end{abstract}

\section{Introduction}

Given a graph $G$ and an integer $t \geq 3$, a $K_{t}$-matching is a set of vertex disjoint copies of $K_{t}$ in $G$. A perfect $K_{t}$-matching (or $K_{t}$-factor) is a spanning $K_{t}$-matching. Clearly, if $G$ contains a perfect $K_{t}$-matching then $t$ divides $|G|$. A classic theorem of Hajnal and Szemerédi [8] states a relationship between the minimum degree and the existence of a perfect $K_{t}$-matching.

Theorem 1.1 (Hajnal-Szemerédi Theorem 8). Let $t>2$ be an integer. Let $G$ be a graph of order $n$ with minimum degree $\delta(G) \geq(t-1) n / t$ and $t \mid n$. Then $G$ contains a perfect $K_{t}$-matching.

*2010 Mathematics Subject Classification: Primary 05C65, 05C70, 05C07. Key words and phrases: Hajnal-Szemerédi, partite, $k$-graph, perfect $K_{k}^{t}$-matching, minimum degree

${ }^{\dagger}$ This author was supported by the ERC, grant no. 258345 . 
Let $G$ be a $t$-partite graph with vertex classes $V_{1}, \ldots, V_{t}$. We say that $G$ is balanced if $\left|V_{i}\right|=\left|V_{j}\right|$ for $1 \leq i<j \leq t$. Write $G\left[V_{i}, V_{j}\right]$ for the induced bipartite subgraph on vertex classes $V_{i}$ and $V_{j}$. Define $\widetilde{\delta}(G)$ to be $\min _{1 \leq i<j \leq t} \delta\left(G\left[V_{i}, V_{j}\right]\right)$. Fischer [6] conjectured the following multipartite version of the Hajnal-Szemerédi theorem.

Conjecture 1.2 (Fischer [6]). Let $G$ be a balanced t-partite graph with each class of size $n$. Then there exists an integer $a_{n, t}$ such that if $\widetilde{\delta}(G) \geq$ $(t-1) n / t+a_{n, t}$, then $G$ contains a perfect $K_{t}$-matching.

Note that the $+a_{n, t}$ term was not presented in Fischer's original conjecture, but it was shown to be necessary for odd $t$ in [19]. For $t=2$, the conjecture can be easily verified by Hall's Theorem. For $t=3$, Johansson 11] proved that $\widetilde{\delta}(G) \geq 2 n / 3+\sqrt{n}$ suffices for all $n$. Using the regularity lemma, Magyar and Martin [19, and Martin and Szemerédi 20] proved Conjecture 1.2 for $t=3$ and $t=4$ respectively for $n$ sufficiently large, where $a_{n, t}=1$ if both $t$ and $n$ are odd, $a_{n, t}=0$ otherwise. For $t \geq 5$, Csaba and Mydlarz 4 proved that $\widetilde{\delta}(G) \geq c_{t} n /\left(c_{t}+1\right)$ is sufficient, where $c_{t}=t-3 / 2+(1+1 / 2+\cdots+1 / t) / 2$. In this paper, we show that Conjecture 1.2 is true asymptotically.

Theorem 1.3. Let $t \geq 2$ be an integer and let $\gamma>0$. Then there exists an integer $n_{0}=n_{0}(t, \gamma)$ such that if $G$ is a balanced $t$-partite graph with each class of size $n \geq n_{0}$ and $\widetilde{\delta}(G) \geq((t-1) / t+\gamma) n$, then $G$ contains a perfect $K_{t}$-matching.

Independently, Theorem 1.3 also has been proved by Keevash and Mycroft [13. Their proof involves the hypergraph blowup lemma [12, so $n_{0}$ is extremely large, whereas our proof gives a much smaller $n_{0}$. Since the submission of this paper, Keevash and Mycroft 14 have proved Conjecture 1.2 provided $n$ is large enough. Also, Han and Zhao 10 gave a different proof of Conjecture 1.2 for $t=3$, again provided $n$ is large enough.

We further generalise Theorem 1.3 to hypergraphs. For $a \in \mathbb{N}$, we refer to the set $\{1, \ldots, a\}$ as $[a]$. For a set $U$, we denote by $\left(\begin{array}{c}U \\ k\end{array}\right)$ the set of $k$-sets of $U$. A $k$-uniform hypergraph, or $k$-graph for short, is a pair $H=$ $(V(H), E(H))$, where $V(H)$ is a finite set of vertices and $E(H) \subset\left(\begin{array}{c}V(H) \\ k\end{array}\right)$ is a family of $k$-sets of $V(H)$. We simply write $V$ to mean $V(H)$ if it is clear from the context. For a $k$-graph $H$ and an $l$-set $T \in\left(\begin{array}{l}V \\ l\end{array}\right)$, let $N^{H}(T)$ be the set of $(k-l)$-sets $S \in\left(\begin{array}{c}V \\ k-l\end{array}\right)$ such that $S \cup T$ is an edge in $H$. Let $\operatorname{deg}^{H}(T)=\left|N^{H}(T)\right|$. Define the minimum l-degree $\delta_{l}(H)$ of $H$ to be the minimal $\operatorname{deg}^{H}(T)$ over all $T \in\left(\begin{array}{c}V \\ l\end{array}\right)$. For $U \subset V$, we denote by $H[U]$ the induced subgraph of $H$ on vertex set $U$.

A $k$-graph $H$ is $t$-partite, if there exists a partition of the vertex set $V$ into $t$ classes $V_{1}, \ldots, V_{t}$ such that every edge intersects every class in at most one vertex. Similarly, $H$ is balanced if $\left|V_{1}\right|=\cdots=\left|V_{t}\right|$. An $l$-set $T \in\left(\begin{array}{c}V \\ l\end{array}\right)$ is said to be legal if $\left|T \cap V_{i}\right| \leq 1$ for $i \in[t]$. For $I \subset[t], T \subset V$ is $I$-legal if $\left|T \cap V_{i}\right|=1$ for $i \in I$ and $\left|T \cap V_{i}\right|=0$ otherwise. We write $V_{I}$ to be the set of $I$-legal sets. For disjoint sets $I, J$ such that $I \cup J \in\left(\begin{array}{c}{[t]} \\ k\end{array}\right)$ and an $I$-legal set $T \in V_{I}$, denote by $N_{J}^{H}(T)$ the set of $J$-legal sets $S$ such that $S \cup T$ is an edge in $H$ and write $\operatorname{deg}_{J}^{H}(T)=\left|N_{J}^{H}(T)\right|$. For $l \in[k-1]$ and $I \in\left(\begin{array}{c}{[t]} \\ l\end{array}\right)$, define $\widetilde{\delta}_{I}(H)=\min \left\{\operatorname{deg}_{J}^{H}(T): T \in V_{I}\right.$ and $\left.J \in\left(\begin{array}{c}{[t] \backslash I} \\ k-|I|\end{array}\right)\right\}$. Finally, we set $\widetilde{\delta}_{l}(H)=\min \left\{\widetilde{\delta}_{I}(H): I \in\left(\begin{array}{c}{[t]} \\ l\end{array}\right)\right\}$. If $H$ is clear from the context, we drop the superscript of $H$. Note that for graphs, when $k=2$, $\widetilde{\delta}_{1}(G)=\widetilde{\delta}(G)$ as defined eariler. 
Let $K_{t}^{k}$ be the complete $k$-graph on $t$ vertices. It is easy to see that a $t$-partite $k$-graph $H$ contains a perfect $K_{t}^{k}$-matching only if $H$ is balanced.

Definition 1.4. Let $1 \leq l<k \leq t$ and $n \geq 1$ be integers. Define $\phi_{l}^{k}(t, n)$ to be the smallest integer $d$ such that every $t$-partite $k$-graph $H$ with each class of size $n$ and $\widetilde{\delta}_{l}(H) \geq d$ contains a perfect $K_{t}^{k}$-matching. Equivalently,

$$
\phi_{l}^{k}(t, n)=\min \left\{d: \widetilde{\delta}_{l}(H) \geq d \Rightarrow H \text { contains a perfect } K_{t}^{k} \text {-matching }\right\},
$$

where $H$ is a t-partite $k$-graph $H$ with each class of size $n$. Write $\phi^{k}(t, n)$ for $\phi_{k-1}^{k}(t, n)$.

Note that Theorem 1.3 implies that $\phi^{2}(t, n) \sim(t-1) n / t$. Various cases of $\phi_{l}^{k}(k, n)$ have been studied. Daykin and Häggkvist [5] showed that $\phi_{1}^{k}(k, n) \leq(k-1) n^{k-1} / k$, which was later improved by Hán, Person and Schacht [9]. Kühn and Osthus [15] showed that $n / 2-1<\phi^{k}(k, n)=$ $\phi_{k-1}^{k}(k, n) \leq n / 2+\sqrt{2 n \log n}$. Aharoni, Georgakopoulos and Sprüssel 1 ] then reduced the upper bound to $\phi^{k}(k, n) \leq\lceil(n+1) / 2\rceil$. For $k / 2 \leq l<$ $k-1$, Pikhurko 21] showed that $\phi_{l}^{k}(k, n) \leq n^{k-l} / 2$. The exact value of $\phi_{1}^{3}(3, n)$ has been determined by the authors in [17. In this paper, we give an upper bound on $\phi^{k}(t, n)$ for $3 \leq k<t$.

Theorem 1.5. For $3 \leq k<t$ and $\gamma \geq 0$, there exists an integer $n_{0}=$ $n_{0}(k, t, \gamma)$ such that for all $n \geq n_{0}$

$$
\phi^{k}(t, n) \leq\left(1-\left(\left(\begin{array}{l}
t-1 \\
k-1
\end{array}\right)+2\left(\begin{array}{l}
t-2 \\
k-2
\end{array}\right)\right)^{-1}+\gamma\right) n .
$$

We do not believe the upper bound is best possible. For $k=3$ and $t=4$, it was shown, independently in [16] and [13], that for any $\gamma>0$ if $H$ is a 3 -graph (not 3-partite) with $\delta_{2}(H)=(3 / 4+\gamma) n$, then $H$ contains a perfect $K_{4}^{3}$-matching, provided $n$ is large enough. (Moreover, in [13, Keevash and Mycroft have determined the exact value of $\delta_{2}(H)$-threshold for the existence of perfect $K_{4}^{3}$-matchings.) Thus, it is natural to believe that $\phi^{3}(4, n)$ should be $3 n / 4+o(n)$.

Our proofs of Theorem 1.3 and Theorem 1.5 use the absorption technique introduced by Rödl, Ruciński and Szemerédi 22. We now present an outline of the absorption technique. First, we remove a set $U$ of disjoint copies of $K_{t}^{k}$ from $H$ satisfying the conditions of the absorption lemma, Lemma 3.2 and call the resulting graph $H^{\prime}$. Next, we find a $K_{t}^{k}$ matching covering almost all vertices of $H^{\prime}$. Let $W$ be the set of 'leftover' vertices. By the absorption property of $U$, there is a perfect $K_{t}^{k}$-matching in $H[U \cup W]$. Hence, we obtain a perfect $K_{t}^{k}$-matching in $H$ as required.

In order to find a $K_{t}^{k}$-matching covering almost all vertices of $H^{\prime}$, we follow the approach of Alon, Frankl, Huang, Rödl, Ruciński and Sudakov [2], who consider fractional matchings. Let $\mathcal{K}_{t}^{k}(H)$ be the set of $K_{t}^{k}$ in a $k$-graph $H$. A fractional $K_{t}^{k}$-matching in a $k$-graph $H$ is a function $w: \mathcal{K}_{t}^{k}(H) \rightarrow[0,1]$ such that for each $v \in V$ we have

$$
\sum\left\{w(T): v \in T \in \mathcal{K}_{t}^{k}(H)\right\} \leq 1 .
$$

Then $\sum_{T \in \mathcal{K}_{t}^{k}(H)} w(T)$ is the size of $w$. If the size is $|H| / t$, then $w$ is perfect. We are interested in perfect fractional $K_{t}^{k}$-matchings $w$ in a $t$ partite $k$-graph $H$ with each class of size $n$. Note that $|H|=t n$, so if $w$ 
is a perfect fractional $K_{t}^{k}$-matching in $H$, then

$$
\sum\left\{w(T): v \in T \in \mathcal{K}_{t}^{k}(H)\right\}=1 \text { for } v \in V \text { and } \sum_{T \in \mathcal{K}_{t}^{k}(H)} w(T)=n .
$$

Define $\phi_{l}^{*, k}(t, n)$ to be the fractional analogue of $\phi_{l}^{k}(t, n)$.

Theorem 1.6. For $2 \leq k \leq t$ and $n \geq 1$,

$$
\lceil(t-k+1) n / t\rceil \leq \phi^{*, k}(t, n) \leq \begin{cases}\lceil(t-1) n / t\rceil & \text { for } k=2, \\
\left\lceil\left(1-\left(\begin{array}{l}
t-1 \\
k-1
\end{array}\right)^{-1}\right) n\right\rceil+1 & \text { for } k \geq 3 .\end{cases}
$$

In particular, $\phi^{*, 2}(t, n)=\lceil(t-1) n / t\rceil$.

Notice that Theorem 1.6 is only tight for $k=2$. The upper bound on $\phi^{*, k}(t, n)$ given in Theorem [1.6] is sufficient for our purpose, that is, to prove Theorem 1.3 and Theorem 1.5. In addition, we also obtain the following result.

Theorem 1.7. Let $2 \leq k \leq t$ be integers. Then, given any $\varepsilon, \gamma>0$, there exists an integer $n_{0}$ such that every $k$-graph $H$ of order $n>n_{0}$ with

$$
\delta_{k-1}(H) \geq t \phi^{*, k}(t,\lceil n / t\rceil)+\gamma n
$$

contains a $K_{t}^{k}$-matching $\mathcal{T}$ covering all but at most $\varepsilon n$ vertices.

Together with Theorem 1.6 we obtain the following corollary for general $k$-graphs.

Corollary 1.8. Let $3 \leq k \leq t$ be integers. Then, given any $\varepsilon, \gamma>0$, there exists an integer $n_{0}$ such that every $k$-graph $H$ of order $n>n_{0}$ with

$$
\delta_{k-1}(H) \geq\left(1-\left(\begin{array}{l}
t-1 \\
k-1
\end{array}\right)^{-1}+\gamma\right) n
$$

contains a $K_{t}^{k}$-matching $\mathcal{T}$ covering all but at most $\varepsilon n$ vertices.

Observe that Corollary 1.8 is a stronger statement than Lemma 6.1 in 16. Thus, by replacing Lemma 6.1 in 16 with Theorem 1.7 we improve the bounds of Theorem 1.4 in 16.

In the next section, we prove Theorem 1.6 Theorem 1.3 and Theorem 1.5 are proved simultaneously in Section 3 Finally, Theorem 1.7 is proved in Section 4

\section{Perfect fractional $K_{t}^{k}$-matchings}

In this section we are going to prove Theorem 1.6. We require Farkas Lemma.

Lemma 2.1 (Farkas Lemma (see 18] P.257)). A system of equations $y A=b, y \geq 0$ is solvable if and only if the system $A x \geq 0, b x<0$ is unsolvable.

First we prove the lower bounds on $\phi^{*, k}(t, n)$.

Proposition 2.2. Let $2 \leq k \leq t$ and $n \geq 1$ be integers. There exists a $t$-partite $k$-graph $H$ with each class of size $n$ with $\widetilde{\delta}_{k-1}(H)=\lceil(t-k+$ 1) $n / t\rceil-1$ without a perfect fractional $K_{t}^{k}$-matching. 
Proof. We fix $t, k$ and $n$. Let $V_{1}, \ldots, V_{t}$ be disjoint vertex sets each of size $n$. For $i \in[t]$, fix a $(\lceil(t-k+1) n / t\rceil-1)$-set $W_{i} \subset V_{i}$. Define $H$ to be the $t$-partite $k$-graph on vertex classes $V_{1}, \ldots, V_{t}$ such that every edge in $H$ meets $W_{i}$ for some $i$. Clearly, $\widetilde{\delta}_{k-1}(H)=\lceil(t-k+1) n / t\rceil-1$. Thus, it suffices to show that $H$ does not contain a perfect fractional $K_{t}^{k}$ matching. Let $A$ be the matrix of $H$ with rows representing the $K_{t}^{k}(H)$ and columns representing the vertices of $H$ such that $A_{T, v}=1$ if and only if $v \in T$ for $T \in \mathcal{K}_{t}^{k}(H)$ and $v \in V$. By Farkas Lemma, Lemma 2.1, taking $y=\left(w(T): T \in \mathcal{K}_{t}^{k}(H)\right)$ and $b=(1, \ldots, 1)$, there is no perfect fractional $K_{t}^{k}$-matching in $H$ if and only if there is a weighting function $w: V \rightarrow \mathbb{R}$ such that

$$
\forall T \in \mathcal{K}_{t}^{k}(H) \sum_{v \in T} w(v) \geq 0 \text { and } \sum_{v \in V} w(v)<0 .
$$

Set $w(v)=(k-1) /(t-k+1)$ if $v \in \bigcup_{i \in[t]} W_{i}$ and $w(v)=-1$ otherwise. Clearly,

$\sum w(v)=\frac{k-1}{t-k+1} t\left(\left\lceil\frac{(t-k+1) n}{t}\right\rceil-1\right)-t\left(n-\left\lceil\frac{(t-k+1) n}{t}\right\rceil+1\right)<0$.

For $T \in \mathcal{K}_{t}^{k}(H), T$ contains at least $t-k+1$ vertices in $\bigcup_{i \in[t]} W_{i}$ and so $\sum_{v \in T} w(v) \geq 0$. Thus, $w$ satisfies (1), so $H$ does not contain a perfect fractional $K_{t}^{k}$-matching.

Proof of Theorem 1.6] By Proposition [2.2, it is sufficient to prove the upper bound on $\phi^{*, k}(t, n)$. Fix $k, t$ and $n$. Suppose the contrary that there exists a $t$-partite $k$-graph $H$ with each class of size $n$ and

$$
\widetilde{\delta}_{k-1}(H) \geq \widetilde{\delta}
$$

that does not contain a perfect fractional $K_{t}^{k}$-matching, where $\widetilde{\delta}$ is the upper bound on $\phi^{*, k}(t, n)$ stated in the theorem. By a similar argument as in the proof of Proposition 2.2 there is a weighting function $w: V \rightarrow \mathbb{R}$ satisfying (10. Let $V_{1}, \ldots, V_{t}$ be the vertex classes of $H$ with $V_{i}=\left\{v_{i, 1}, \ldots, v_{i, n}\right\}$ for $i \in[t]$. We identify the $t$-tuple $\left(j_{1}, \ldots, j_{t}\right) \in[n]^{t}$ with the [t]-legal set $\left\{v_{1, j_{1}}, \ldots, v_{t, j_{t}}\right\}$ and write $w\left(j_{1}, \ldots, j_{t}\right)$ to mean $\sum_{i \in[t]} w\left(v_{i, j_{i}}\right)$. Without loss of generality we may assume that for $i \in[t]$, $\left(w\left(v_{i, j}\right)\right)_{j \in[n]}$ is a decreasing sequence, i.e. $w\left(v_{i, j}\right) \geq w\left(v_{i, j^{\prime}}\right)$ for $1 \leq j<$ $j^{\prime} \leq n$. By considering the vertex weighting $w^{\prime}$ such that

$$
w^{\prime}(v)= \begin{cases}w(v)+\varepsilon & \text { if } v \in V_{i} \\ w(v)-\varepsilon & \text { if } v \in V_{i^{\prime}} \\ w(v) & \text { otherwise }\end{cases}
$$

with $\varepsilon>0$, we may assume that $w\left(v_{i, n}\right)=w\left(v_{i^{\prime}, n}\right)$ for all $i, i^{\prime} \in[t]$. By (1), $w\left(v_{i, n}\right)$ is negative as $w\left(v_{i, j}\right) \geq w\left(v_{i, n}\right)=w\left(v_{i^{\prime}, n}\right)$ for all $j \in[n]$ and $i, i^{\prime} \in[t]$. Thus, by multiplying through by a suitable constant we may assume that $w\left(v_{i, n}\right)=-1$ for all $i \in[t]$. We further assume that $w(v) \leq t-1$ for all $v \in V$, because (1) still holds after we replace $w(v)$ with $\min \{w(v), t-1\}$. Finally, we apply the linear transformation $(w(v)+1) / t$ for $v \in V$, which scales $w$ so that it now lies in the interval $[0,1]$ and $w$ satisfies the following inequalities

$$
\forall T \in \mathcal{K}_{t}^{k}(H) \sum_{v \in T} w(v) \geq 1 \text { and } \sum_{v \in V} w(v)<n .
$$


For $j \in[t]$, set $r(j)=n-\left(\begin{array}{c}j-1 \\ k-1\end{array}\right)(n-\widetilde{\delta})$. Given a $J$-legal set $T \in \mathcal{K}_{j}^{k}(H)$ with $J \in\left(\begin{array}{c}{[t]} \\ j\end{array}\right)$ and $j<k$, for each $i \in[t] \backslash J$ there are at least $r(j+1)$ vertices $v \in V_{i}$ such that $T \cup v$ forms a $K_{j+1}^{k}$. Note that $r(j)=n$ for $j \in[k-1]$ and $r(k)=\widetilde{\delta}$. By the definition of $\widetilde{\delta}$, we know that $r(t) \geq 1$. Hence, we can find a $K_{t}^{k}\left(j_{1}, j_{2}, \ldots, j_{t}\right)$ with $j_{i} \geq r(i)$ for $i \in[t]$. fore,

Recall that for $i \in[t]$ and $1 \leq j<j^{\prime} \leq n, w\left(v_{i, j}\right) \geq w\left(v_{i, j^{\prime}}\right)$. There-

$$
\sum_{i \in[t]} w\left(v_{i, r(i)}\right)=w(r(1), r(2), \ldots, r(t)) \geq w\left(j_{1}, j_{2}, \ldots, j_{t}\right) \geq 1
$$

by (2). By a similar argument, for any permutation $\sigma$ of $[t]$ we have

$$
\sum_{i \in[t]} w\left(v_{i, r(\sigma(i))}\right) \geq 1 .
$$

Setting $\sigma=(1,2, \ldots, t)$, we have

$$
\sum_{i \in[t]} \sum_{j \in[t]} w\left(v_{i, r(j)}\right)=\sum_{j \in[t]} \sum_{i \in[t]} w\left(v_{i, r\left(\sigma^{j}(i)\right)}\right) \geq t .
$$

Observe that $w\left(v_{i, r(j)}\right) \leq w\left(v_{i, r(j+1)}\right)$ for $i \in[t]$ and $j \in[t-1]$. Since $r(j)=n$ for $j \in[k-1]$ and $w\left(v_{i, n}\right)=0$ for $i \in[t]$,

$$
\begin{aligned}
\sum_{i \in[t]} w\left(v_{i, r(t)}\right) & =\frac{1}{t-k+1} \sum_{i \in[t]}\left(\sum_{j \in[k-1]} w\left(v_{i, r(j)}\right)+(t-k+1) w\left(v_{i, r(t)}\right)\right) \\
& \geq \frac{1}{t-k+1} \sum_{i \in[t]} \sum_{j \in[t]} w\left(v_{i, r(j)}\right) \geq \frac{t}{t-k+1}
\end{aligned}
$$

where the last inequality is due to (3).

Claim 2.3.

$\sum_{i \in[t]}\left(\sum_{j \in[t-1]}(r(j)-r(j+1)) w\left(v_{i, r(j)}\right)+\frac{r(k)-r(t)}{t-k} w\left(v_{i, r(t)}\right)\right) \geq \frac{t(r(k)-r(t))}{t-k}$.

Proof of claim. Consider the multiset $A$ containing $(t-k)(r(j)-r(j+1))$ copies of $j$ for $k \leq j \leq t-1$ and $r(k)-r(t)$ copies of $t$. In order to prove the claim, (by multiplying though by $(t-k)$ ), it is enough to show that

$$
\sum_{i \in[t]} \sum_{j \in A} w\left(v_{i, r(j)}\right) \geq t(r(k)-r(t)) .
$$

First note that

$$
\sum_{k \leq j \leq t-1}(r(j)-r(j+1))=r(k)-r(t),
$$

so the number of elements $j$ (with multiplicity) in $A$ with $k \leq j \leq t-1$ is exactly $(t-k)(r(k)-r(t))$. Note that $r(j)-r(j+1)=\left(\begin{array}{c}j-1 \\ k-2\end{array}\right)(n-\widetilde{\delta})$. Hence, for $k \leq j<j^{\prime} \leq t-1$, there are more copies of $j^{\prime}$ than copies of $j$ in $A$. Recall that $A$ contains precisely $r(k)-r(t)$ copies of $t$. It follows that we can replace some elements by smaller elements to obtain a multiset $A^{\prime}$ containing each of $k, \ldots, t$ exactly $r(k)-r(t)$ times. Since 
$w\left(v_{i, r(j)}\right)$ is increasing in $j$ and $w\left(v_{i, r(j)}\right)=0$ for $j \in[k-1]$, it follows that

$$
\begin{aligned}
\sum_{i \in[t]} \sum_{j \in A} w\left(v_{i, r(j)}\right) & \geq \sum_{i \in[t]} \sum_{j \in A^{\prime}} w\left(v_{i, r(j)}\right)=(r(t)-r(k)) \sum_{i \in[t]} \sum_{k \leq j \leq t} w_{v_{i, r(j)}} \\
& =(r(t)-r(k)) \sum_{i \in[t]} \sum_{j \in[t]} w\left(v_{i, r(j)}\right) \geq t(r(t)-r(k))
\end{aligned}
$$

as required, where the last inequality is due to (3).

Recall that $r(k)=\widetilde{\delta}$ and $r(1)=n$. Since $w\left(v_{i, j^{\prime}}\right)$ is decreasing in $j^{\prime}$, $w\left(v_{i, j^{\prime}}\right) \geq w\left(v_{i, r(j)}\right)$ for $r(j+1)<j^{\prime} \leq r(j)$ and $j \in[t]$, where we take $r(t+1)=0$. Hence,

$$
\sum_{i \in[t]} \sum_{j \in[n]} w\left(v_{i, j}\right) \geq \sum_{i \in[t]}\left(\sum_{j \in[t-1]}(r(j)-r(j+1)) w\left(v_{i, r(j)}\right)+r(t) w\left(v_{i, r(t)}\right)\right) .
$$

By Claim 2.3 and (4), this is at least

$$
\begin{aligned}
& \frac{t(r(k)-r(t))}{t-k}+\sum_{i \in[t]}\left(r(t)-\frac{r(k)-r(t)}{t-k}\right) w\left(v_{i, r(t)}\right) \\
\geq & \frac{t(r(k)-r(t))}{t-k}+\left(r(t)-\frac{r(k)-r(t)}{t-k}\right) \frac{t}{t-k+1} \\
= & \frac{t r(k)}{t-k+1}=\frac{t \tilde{\delta}}{t-k+1} \geq n
\end{aligned}
$$

contradicting (2). The proof of Theorem 1.6 is completed.

Note that the inequality above suggests that for $k \geq 3$, we would have $\phi^{*, k}(t, n)=\widetilde{\delta} \leq\lceil(t-k+1) n / t\rceil$. However, our proof requires that $1 \leq r(t)=n-\left(\begin{array}{c}t-1 \\ k-1\end{array}\right)(n-\widetilde{\delta})$ implying that $\widetilde{\delta} \geq\left(1-\left(\begin{array}{c}t-1 \\ k-1\end{array}\right)^{-1}\right) n+1$.

\section{Proof of Theorem 1.3 and Theorem 1.5}

First we need the following simple proposition.

Proposition 3.1. Let $\gamma>0$. Let $H$ be a balanced t-partite $k$-graph with partition classes $V_{1}, \ldots, V_{t}$, each of size $n$ with

$$
\widetilde{\delta}_{k-1}(H) \geq\left(1-\left(\left(\begin{array}{l}
t-2 \\
k-1
\end{array}\right)+2\left(\begin{array}{l}
t-2 \\
k-2
\end{array}\right)\right)^{-1}+\gamma\right) n .
$$

Then, for $i \in[t]$ and distinct vertices $u, v \in V_{i}$, there are at least $(\gamma n)^{t-1}$ legal $[t] \backslash i$-sets $T$ such that $T \cup u$ and $T \cup v$ span copies of $K_{t}^{k}$ in $H$.

Proof. Let $u, v \in V_{1}$. For $2 \leq i \leq t$, we pick $w_{i} \in V_{i}$ such that $w_{i} \in N(T)$ for all legal $(k-1)$-sets $T \subset\left\{u, v, w_{2}, \ldots, w_{i-1}\right\}$. By the definition of $\widetilde{\delta}_{k-1}(H)$, there are at least $\gamma n$ choices for each $w_{i}$. The proposition easily follows.

Using Proposition 3.1 we obtain an absorption lemma. Its proof can be easily obtained by modifying the proof of Lemma 4.2 in [17]. For the sake of completeness, it is included in Appendix A. 
Lemma 3.2 (Absorption lemma). Let $2 \leq k<t$ be integers and let $\gamma>0$. Then, there is an integer $n_{0}$ satisfying the following: for each balanced $t$-partite $k$-graph $H$ with each class of size $n \geq n_{0}$ and

$$
\widetilde{\delta}_{k-1}(H) \geq\left(1-\left(\left(\begin{array}{c}
t-2 \\
k-1
\end{array}\right)+2\left(\begin{array}{c}
t-2 \\
k-2
\end{array}\right)\right)^{-1}+\gamma\right) n
$$

there exists a balanced vertex subset $U \subset V(H)$ of size $|U| \leq \gamma^{t(t-1)} n /\left(t^{2} 2^{t+2}\right)$ such that there exists a perfect $K_{t}^{k}$-matching in $H[U \cup W]$ for every balanced vertex subset $W \subset V \backslash U$ of size $|W| \leq \gamma^{2 t(t-1)} n /\left(t^{2} 2^{2 t+5}\right)$.

Our next task is to find a large $K_{t}^{k}$-matching in $H$ covering all but at most $\varepsilon n$ vertices, which requires a theorem of Frankl and Rödl [7] and Chernoff's inequality. The proof of Lemma 3.5 is based on Claim 4.1 in 2]. For constants $a, b, c>0$, write $a=b \pm c$ for $b-c \leq a \leq b+c$.

Theorem 3.3 (Frankl and Rödl [7]). For all $t, \varepsilon \geq 0$ and $a>3$, there exists $\tau=\tau(\varepsilon), D=D(n)$, and $n_{0}=n_{0}(\tau)$ such that if $n \geq n_{0}$ and $H$ is a t-graph of order $n$ satisfying

1. $\operatorname{deg}^{H}(v)=(1 \pm \tau) D$ for all $v \in V$, and

2. $\Delta_{2}(H)=\max _{T \in\left(\begin{array}{c}V(H) \\ 2\end{array}\right)} \operatorname{deg}^{H}(T)<D /(\log n)^{a}$

then $H$ contains a matching $M$ covering all but at most $\varepsilon n$ vertices.

Lemma 3.4 (Chernoff's inequality (see e.g. [3])). Let $X \sim \operatorname{Bin}(n, p)$. Then, for $0<\lambda \leq n p$

$\mathbb{P}(|X-n p| \geq \lambda) \leq 2 \exp \left(-\frac{\lambda^{2}}{4 n p}\right)$ and $\mathbb{P}(X \leq n p-\lambda) \leq \exp \left(-\frac{\lambda^{2}}{4 n p}\right)$.

Lemma 3.5. Let $2 \leq k \leq t$ be integers. Then, for any given $\varepsilon, \gamma>0$, there exists an integer $n_{0}$ such that every $t$-partite $k$-graph $H$ with partition classes $V_{1}, \ldots, V_{t}$, each of size $n>n_{0}$, with

$$
\widetilde{\delta}_{k-1}(H) \geq \phi^{*, k}(t, n)+\gamma n
$$

contains a $K_{t}^{k}$-matching $\mathcal{T}$ covering all but at most $\varepsilon n$ vertices.

Proof. Fix $k, t$ and $\varepsilon$. If $k=t=2$, then the lemma easily holds and so we may assume that $t \geq 3$. Write $\phi^{*}=\phi^{*, k}(t, n) / n$. We assume that $n$ is sufficiently large throughout the proof. Let $H$ be a balanced $t$-partite $k$-graph $H$ with partition classes $V_{1}, \ldots, V_{t}$, each of size $n$, with $\widetilde{\delta}_{k-1}(H) \geq\left(\phi^{*}+\gamma\right) n$. Our aim is to define a $t$-graph $H^{*}$ on vertex set $V(H)$ satisfying the condition of Theorem 3.3 where every edge in $H^{*}$ corresponds to a $K_{t}^{k}$ in $H$. Hence, by Theorem 3.3 , there exists a matching $M$ covering all but at most $\varepsilon n$ vertices of $H^{*}$ corresponding to a $K_{t}^{k}$-matching in $H$.

We are going to construct $H^{*}$ via two rounds of randomisation. For $i \in$ [t], let $R_{i}$ be a random binomial subset of $V_{i}$ with probability $p=n^{-0.9}$. Let $R=\left(R_{1}, \ldots, R_{t}\right)$. Then, by Chernoff's inequality (Lemma 3.4)

$$
\mathbb{P}\left(\left|R_{i}-n^{0.1}\right| \geq n^{0.075}\right) \leq 2 \exp \left(-n^{0.05} / 2\right) .
$$

For each $I \in\left(\begin{array}{c}{[t]} \\ k-1\end{array}\right)$, each $I$-legal set $T \subset R$ and $i \in[t] \backslash I$

$$
\mathbb{E}\left(\operatorname{deg}_{i}^{H[R]}(T)\right) \geq\left(\phi^{*}+\gamma\right) n \times n^{-0.9}=\left(\phi^{*}+\gamma\right) n^{0.1} .
$$


Again, by Chernoff's inequality (Lemma 3.4)

$\mathbb{P}\left(\operatorname{deg}_{i}^{H[R]}(T)<\left(\phi^{*}+\gamma / 2\right) n^{0.1}\right) \leq \exp \left(-\gamma^{2} n^{0.1} /\left(16\left(\phi^{*}+\gamma\right)\right)\right)=e^{-\Omega\left(n^{0.1}\right)}$.

Let $m=n^{0.1}-n^{0.075}$. Let $R_{i}^{\prime}$ be a randomly chosen $m$-set in $R_{i}$ and let $R^{\prime}=\left(R_{1}^{\prime}, \ldots, R_{t}^{\prime}\right)$. By (5) and (6) , we have with probability $1-e^{-\Omega\left(n^{0.05}\right)}$

$$
\widetilde{\delta}_{k-1}\left(H\left[R^{\prime}\right]\right) \geq(c+\gamma / 2) n^{0.1}-2 n^{0.075} \geq(c+\gamma / 4) m .
$$

Since $R_{i}^{\prime}$ is chosen randomly from $R_{i}$, which is also chosen randomly, a given element is chosen in $R_{i}^{\prime}$ with probability $m / n=n^{-0.9}-n^{-0.925}$ minus an exponentially small correction term. Hence we may assume that for $v \in V$

$$
n^{-0.9} \geq \mathbb{P}\left(v \in R^{\prime}\right) \geq\left(1-2 n^{-0.025}\right) n^{-0.9} .
$$

Now, we take $n^{1.1}$ independent copies of $R^{\prime}$ and denote them by $R^{\prime}(1)$, $R^{\prime}(2), \ldots, R^{\prime}\left(n^{1.1}\right)$. For a subset of vertices $S \subset V$, let

$$
Y_{S}=\left|\left\{i: S \subset R^{\prime}(i)\right\}\right| \text {. }
$$

Since the probability that a particular $R^{i}$ (not $R^{\prime}(i)$ ) contains $S$ is $n^{-0.9 n}$, $\mathbb{E}\left(Y_{S}\right) \leq n^{1.1-0.9|S|}$. With probability at least $1-2 \exp \left(-9 n^{1.5} / 2\right)$ by Lemma 3.4 $Y_{v}=n^{0.2} \pm 3 n^{0.175}$ for every $v \in V$, where recall that $y=x \pm c$ means $x-c \leq y \leq x+c$. Let $Z_{2}=\left|\left\{S \in\left(\begin{array}{c}V \\ 2\end{array}\right): Y_{S} \geq 3\right\}\right|$ and observe that

$$
\mathbb{E}\left(Z_{2}\right)<n^{2}\left(n^{1.1}\right)^{3}\left(n^{-0.9}\right)^{6}=n^{-0.1} .
$$

Let $Z_{3}=\left|\left\{S \in\left(\begin{array}{c}V \\ 3\end{array}\right): Y_{S} \geq 2\right\}\right|$ and observe that

$$
\mathbb{E}\left(Z_{3}\right)<n^{3}\left(n^{1.1}\right)^{2}\left(n^{-0.9}\right)^{6}=n^{-0.2} .
$$

The latter implies that every 3 -set $S \in\left(\begin{array}{l}V \\ 3\end{array}\right)$ lies in at most one $R^{\prime}(i)$ with high probability. In summary, there exist $n^{1.1}$ vertex sets $R^{\prime}(1), \ldots$, $R^{\prime}\left(n^{1.1}\right)$ such that

(i) for every $v \in V, Y_{v}=n^{0.2} \pm 3 n^{0.175}$,

(ii) every 2-set $S \in\left(\begin{array}{c}V \\ 2\end{array}\right)$ is in at most two sets $R^{\prime}(i)$,

(iii) every 3-set $S \in\left(\begin{array}{l}V \\ 3\end{array}\right)$ is in at most one set $R^{\prime}(i)$,

(iv) for $i \in\left[n^{1.1}\right], R^{\prime}(i)=\left(R_{1}^{\prime}, \ldots R_{t}^{\prime}\right)$ with $R_{j}^{\prime} \subset V_{j}$ and $\left|R_{j}^{\prime}\right|=m$ for $j \in[t]$,

(v) for $i \in\left[n^{1.1}\right], \widetilde{\delta}_{k-1}\left(H\left[R^{\prime}(i)\right]\right) \geq\left(\phi^{*}+\gamma / 4\right) m$.

Fix one such sequence $R^{\prime}(1), \ldots, R^{\prime}\left(n^{1.1}\right)$.

By $(v)$ and the definition of $\phi^{*}$, there exists a fractional perfect $K_{t}^{k}$ matching $w^{i}$ in $H\left[R^{\prime}(i)\right]$ for $i \in\left[n^{1,1}\right]$. Now we conduct our second round of random process by defining a random $t$-graph $H^{*}$ on vertex classes $V$ such that each $[t]$-legal set $T$ is randomly independently chosen with

$$
\mathbb{P}\left(T \in H^{*}\right)= \begin{cases}w^{i_{T}}(T) & \text { if } T \in \mathcal{K}_{t}^{k}\left(H\left[R^{\prime}\left(i_{T}\right)\right]\right) \text { for some } i_{T} \in[t], \\ 0 & \text { otherwise }\end{cases}
$$

Note that $i_{T}$ is unique by (iii) (as $t \geq 3$ ) and so $H^{*}$ is well defined. For $v \in V$, let $I_{v}=\left\{i: v \in R^{\prime}(i)\right\}$ and so $\left|I_{v}\right|=Y_{v}=n^{0.2} \pm 3 n^{0.175}$ by $(i)$. For every $v \in V$, let $E_{v}^{i}$ be the set of $K_{t}^{k}$ in $H\left[R^{\prime}(i)\right]$ containing $v$. 
Thus, for $v \in V$, $\operatorname{deg}^{H^{*}}(v)$ is a generalised binomial random variable with expectation

$$
\mathbb{E}\left(\operatorname{deg}^{H^{*}}(v)\right)=\sum_{i \in I_{v}} \sum_{T \in E_{v}^{i}} w^{i}(T)=\left|I_{v}\right|=n^{0.2} \pm 3 n^{0.175} .
$$

Similarly, for every 2-set $\{u, v\}$,

$$
\mathbb{E}\left(\operatorname{deg}^{H^{*}}(u, v)\right)=\sum_{i \in I_{v} \cap I_{u}} \sum_{T \in E_{v}^{i} \cap E_{u}^{i}} w^{i}(T) \leq\left|I_{v} \cap I_{u}\right| \leq 2,
$$

by (ii). Hence, again by Chernoff's inequality, Lemma 3.4 we may assume that for every $v \in V$ and every 2 -set $\{u, v\}$

$$
\operatorname{deg}^{H^{*}}(v)=n^{0.2} \pm 4 n^{0.2-\varepsilon}, \quad \operatorname{deg}^{H^{*}}(u, v)<n^{0.1} .
$$

Thus, $H^{*}$ satisfies the hypothesis of Theorem 3.3 and the proof is completed.

Next we prove Theorem 1.3 and Theorem 1.5

Proof of Theorem 1.3 and Theorem 1.5. Fix $k$ and $t$ and $\gamma>0$. Let

$$
d= \begin{cases}(t-1) n / t & \text { if } k=2 \\
\left(1-\left(\left(\begin{array}{l}
t-1 \\
k-1
\end{array}\right)+2\left(\begin{array}{l}
t-2 \\
k-2
\end{array}\right)\right)^{-1}\right) n & \text { if } k \geq 3\end{cases}
$$

Note that $d \geq \phi^{*, k}(t, n)$ by Theorem [1.6. Let $H$ be a $t$-partite $k$-graph with vertex classes $V_{1}, \ldots, V_{t}$ each of size $n \geq n_{0}$ and $\widetilde{\delta}_{k-1}(H) \geq d+\gamma n$. We are going to show that $H$ contains a perfect $K_{t}^{k}$-matching. Throughout this proof, $n_{0}$ is assumed to be sufficiently large. By Lemma 3.2 there exists a balanced vertex set $U$ in $V$ of size $|U| \leq \gamma^{t(t-1)} n /\left(t^{2} 2^{t+2}\right)$ such that there exists a perfect $K_{t}^{k}$-matching in $H[U \cup W]$ for every balanced vertex subset $W \subset V \backslash U$ of size $|W| \leq \gamma^{2 t(t-1)} n /\left(t^{2} 2^{2 t+5}\right)$. Set $H^{\prime}=$ $H[V \backslash U]$ and note that $\widetilde{\delta}_{k-1}\left(H^{\prime}\right) \geq d+\gamma n / 2 \geq\left(\phi^{*, k}(t, n)+\gamma / 2\right) n$. By Lemma 3.5 there exists a $K_{t}^{k}$-matching $\mathcal{T}$ in $H^{\prime}$ covering all but at most $\varepsilon n$ vertices of $H^{\prime}$, where $\varepsilon=\gamma^{2 t(t-1)} /\left(t^{2} 2^{2 t+5}\right)$. Let $W=V\left(H^{\prime}\right) \backslash V(\mathcal{T})$, so $W$ is balanced. Since $H[U \cup W]$ contains a perfect $K_{t}^{k}$-matching $\mathcal{T}^{\prime}$ by the choice of $U, \mathcal{T} \cup \mathcal{T}^{\prime}$ is a perfect $K_{t}^{k}$-matching in $H$.

\section{Proof of Theorem 1.7}

Note that together Lemma 3.5 and the lemma below imply Theorem 1.7 Hence all that remains is to prove Lemma 4.1 .

Lemma 4.1. For integers $t \geq k \geq 2$, there exists $n_{0}$ such that the following holds. Suppose that $H$ is a $k$-graph with $n \geq n_{0}$ vertices with $t \mid n$. Then there exists a partition $V_{1}, \ldots, V_{t}$ of $V(H)$ into sets of size $n / t$ such that for every $l \in[k-1]$, every $I \in\left(\begin{array}{c}{[t]} \\ l\end{array}\right)$, every legal $I$-set $T$ and $J \in\left(\begin{array}{c}{[t] \backslash I} \\ k-l\end{array}\right)$, we have

$$
\frac{t^{k-l}}{(k-l) !} \operatorname{deg}_{J}^{H^{\prime}}(T) \geq \operatorname{deg}^{H}(T)-2(t \ln n)^{1 / 2} n^{k-l-1 / 2},
$$

where $H^{\prime}$ is the induced $t$-partite $k$-subgraph of $H$ with vertex classes $V_{1}$, $\ldots, V_{t}$. 
Proof. First set $m=k-l$ and let $U_{1}, \ldots, U_{t}$ be a random partition of $V$, where each vertex appears in vertex class $U_{j}$ independently with probability $1 / t$. For a fixed $l$-set $T=\left\{v_{1}, \ldots, v_{l}\right\}$, let $N^{H}(T)$ be the link hypergraph of $T$. Thus, $N^{H}(T)$ is an $m$-graph with $\operatorname{deg}^{H}(T)$ edges. We decompose $N^{H}(T)$ into $i_{0} \leq m n^{m-1}$ nonempty pairwise edge disjoint matchings, which we denote by $M_{1}, \ldots, M_{i_{0}}$. To see that this is possible consider the auxiliary graph $G$ with $V(G)=E\left(N^{H}(T)\right)$, in which for $A, B \in N^{H}(T) A$ and $B$ are joined in $G$ if and only if $A \cap B \neq \emptyset$. Since $G$ has maximum degree at most $m\left(\begin{array}{c}n-1 \\ m-1\end{array}\right), G$ can be properly coloured using at most $m n^{m-1}$ colours, where each colour class corresponds to a matching.

For every edge $E \in N^{H}(T)$, and every index set $J \in\left(\begin{array}{c}{[t]} \\ m\end{array}\right)$, we say that $E$ is $J$-good, if $E$ is $J$-legal with respect to $U_{1}, \ldots, U_{t}$. Since the partition $U_{1}, \ldots, U_{t}$ was chosen randomly, we have for fixed $J \in\left(\begin{array}{c}{[t]} \\ m\end{array}\right)$

$$
\mathbb{P}(E \text { is } J \text {-good })=m ! t^{-m} .
$$

Thus, for $X_{i, J}=X_{i, J}(T)=\mid\left\{E \in M_{i}: E\right.$ is $J$-good $\} \mid$ we have

$$
\mu_{i, J}=\mu_{i, J}(T)=\mathbb{E}\left(X_{i, J}\right)=\frac{m !}{t^{m}}\left|M_{i}\right| .
$$

Now call a matching $M_{i}$ bad (with respect to $U_{1}, \ldots, U_{t}$ ) if there exists a set $J \in\left(\begin{array}{c}{[t]} \\ m\end{array}\right)$ such that

$$
X_{i, J} \leq\left(1-\left(\frac{2(2 k-1) \ln n}{\mu_{i, J}}\right)^{1 / 2}\right) \mu_{i, J}
$$

and call $T$ a bad set if there is at least one bad $M_{i}=M_{i}(T)$. Otherwise call $T$ a good set. For a fixed $M_{i}$ the events ' $E$ is $J$-good' with $E \in M_{i}$ are jointly independent, hence by Chernoff's inequality, Lemma 3.4

$$
\mathbb{P}\left(M_{i} \text { is bad }\right) \leq\left(\begin{array}{c}
t \\
m
\end{array}\right) \exp (-(2 k-1) \ln n)=\left(\begin{array}{c}
t \\
m
\end{array}\right) n^{-2 k+1} .
$$

Recall that $i_{0} \leq m n^{m-1}$ and $m \leq k-1$, we have

$$
\mathbb{P}(T \text { is bad }) \leq i_{0}\left(\begin{array}{c}
t \\
m
\end{array}\right) n^{-2 k+1} \leq n^{-k}
$$

and by summing over all $l$-sets $T$ we obtain that

$$
\mathbb{P}(\text { there exists a bad } l \text {-set }) \leq n^{-1} .
$$

Moreover, Chernoff's inequality, Lemma 3.4 yields

$$
\mathbb{P}\left(\left|U_{j}\right| \geq n / t+n^{1 / 2}(\ln n)^{1 / 4} / t\right) \leq \exp \left(-(\ln n)^{1 / 2} / 4 t\right) .
$$

Thus with positive probability there is a partition $U_{1}, \ldots, U_{t}$ such that all $l$-sets $T$ are good and

$$
\left|U_{j}\right| \leq n / t+n^{1 / 2}(\ln n)^{1 / 4} / t \text { for all } j \in[t] .
$$

Consequently, by redistributing at most $n^{1 / 2}(\ln n)^{1 / 4}$ vertices of the partition $U_{1}, \ldots, U_{t}$ we obtain an equipartition $V_{1}, \ldots, V_{t}$ with

$$
\left|V_{j}\right|=n / t \text { and }\left|U_{j} \backslash V_{j}\right| \leq n^{1 / 2}(\ln n)^{1 / 4} / t \text { for all } j \in[t] .
$$


Let $H^{\prime}$ be the induced $t$-partite $k$-subgraph with vertex classes $V_{1}, \ldots, V_{t}$. Note that for an $l$-set $I \in\left(\begin{array}{c}{[t]} \\ l\end{array}\right)$, a $I$-legal set $T$ and an $m$-set $J \in\left(\begin{array}{c}{[t] \backslash I} \\ m\end{array}\right)$,

$$
\begin{aligned}
\operatorname{deg}_{J}^{H^{\prime}}(T) & \geq \sum_{i \in\left[i_{0}\right]}\left(1-\left(\frac{2(2 k-1) \ln n}{\mu_{i, J}}\right)\right) \mu_{i, J}-m \frac{n^{1 / 2}(\ln n)^{1 / 4}}{t} n^{m-1} \\
& \geq \frac{m !}{t^{m}} \operatorname{deg}_{J}^{H}(T)-(2(2 k-1) \ln n)^{1 / 2} \sum_{i \in\left[i_{0}\right]} \mu_{i, J}^{1 / 2}-m \frac{n^{1 / 2}(\ln n)^{1 / 4}}{t} n^{m-1} .
\end{aligned}
$$

By the Cauchy-Schwarz inequality, we obtain that

$$
\sum_{i \in\left[i_{0}\right]} \mu_{i, J}^{1 / 2} \leq\left(i_{0} \sum_{i \in\left[i_{0}\right]} \mu_{i, J}\right)^{1 / 2} \leq\left(m n^{m-1} \frac{m !}{t^{m}}\left(\begin{array}{c}
n \\
m
\end{array}\right)\right)^{1 / 2} \leq n^{m-1 / 2}
$$

Therefore,

$$
\operatorname{deg}_{J}^{H}(T) \geq \frac{m !}{t^{m}} \operatorname{deg}_{J}^{H}(T)-2(k \ln n)^{1 / 2} n^{m-1 / 2},
$$

as required.

\section{Acknowledgment}

The authors would like to thank the anonymous referee for the helpful comments.

\section{References}

[1] R. Aharoni, A. Georgakopoulos, and P. Sprüssel, Perfect matchings in r-partite r-graphs, European J. Combin. 30 (2009), no. 1, 39-42.

[2] N. Alon, P. Frankl, H. Huang, V. Rödl, A. Ruciński, and B. Sudakov, Large matchings in uniform hypergraphs and the conjectures of Erdös and Samuels, J. Combin. Theory Ser. A 119 (2012), no. 6, 1200-1215.

[3] N. Alon and J. H. Spencer, The probabilistic method, second ed., Wiley-Interscience Series in Discrete Mathematics and Optimization, Wiley-Interscience [John Wiley \& Sons], New York, 2000, With an appendix on the life and work of Paul Erdős.

[4] B. Csaba and Mydlarz M., Approximate multipartite version of the Hajnal-Szemerédi theorem, J. Combin. Theory Ser. B 102 (2012), no. 2, 395-410.

[5] D. E. Daykin and R. Häggkvist, Degrees giving independent edges in a hypergraph, Bull. Austral. Math. Soc. 23 (1981), no. 1, 103-109.

[6] E. Fischer, Variants of the Hajnal-Szemerédi theorem, J. Graph Theory 31 (1999), no. 4, 275-282.

[7] P. Frankl and V. Rödl, Near perfect coverings in graphs and hypergraphs, European J. Combin. 6 (1985), no. 4, 317-326.

[8] A. Hajnal and E. Szemerédi, Proof of a conjecture of P. Erdös, Combinatorial theory and its applications, II (Proc. Colloq., Balatonfüred, 1969), North-Holland, Amsterdam, 1970, pp. 601-623.

[9] H. Hàn, Y. Person, and M. Schacht, On perfect matchings in uniform hypergraphs with large minimum vertex degree, SIAM J. Discrete Math. 23 (2009), no. 2, 732-748. 
[10] J. Han and Y. Zhao, On multipartite Hajnal-Szemerédi theorems, Arxiv preprint arXiv:1203.2667 (2012).

[11] R. Johansson, Triangle-factors in a balanced blown-up triangle, Discrete Math. 211 (2000), no. 1-3, 249-254.

[12] P. Keevash, A hypergraph blow-up lemma, Random Structures \& Algorithms 39 (2011), no. 3, 275-376.

[13] P. Keevash and R. Mycroft, A geometric theory for hypergraph matching, Arxiv preprint arXiv:1108.1757 (2011).

[14] P. Keevash and R. Mycroft, A multipartite Hajnal-Szemerédi theorem, Arxiv preprint arXiv:1201.1882 (2012).

[15] D. Kühn and D. Osthus, Matchings in hypergraphs of large minimum degree, J. Graph Theory 51 (2006), no. 4, 269-280.

[16] A. Lo and K. Markström, F-factors in hypergraphs via absorption, Arxiv preprint arXiv:1105.3411 (2011).

[17] A. Lo and K. Markström, Perfect matchings in 3-partite 3-uniform hypergraphs, Arxiv preprint arXiv:1103.5654 (2011).

[18] L. Lovász and M. D. Plummer, Matching theory, North-Holland Mathematics Studies, vol. 121, North-Holland Publishing Co., Amsterdam, 1986, Annals of Discrete Mathematics, 29.

[19] C. Magyar and R. Martin, Tripartite version of the Corrádi-Hajnal theorem, Discrete Math. 254 (2002), no. 1-3, 289-308.

[20] R. Martin and E. Szemerédi, Quadripartite version of the HajnalSzemerédi theorem, Discrete Math. 308 (2008), no. 19, 4337-4360.

[21] O. Pikhurko, Perfect matchings and $K_{4}^{3}$-tilings in hypergraphs of large codegree, Graphs Combin. 24 (2008), no. 4, 391-404.

[22] V. Rödl, A. Ruciński, and E. Szemerédi, Perfect matchings in large uniform hypergraphs with large minimum collective degree, J. Combin. Theory Ser. A 116 (2009), no. 3, 613-636.

\section{A Proof of Lemma 3.2}

Proof. Throughout the proof we may assume that $n_{0}$ is chosen sufficiently large. Let $H$ be a balanced $t$-partite $k$-graph with partition classes $V_{1}$, $\ldots, V_{t}$ each of size $n$ and $\widetilde{\delta}_{k-1}(H) \geq \widetilde{\delta}$, where $\widetilde{\delta}$ is the lower bound on $\widetilde{\delta}_{k-1}(H)$ stated in the lemma. Let $H^{\prime}$ be the $t$-partite $t$-graph on $V_{1}, \ldots$, $V_{t}$ in which $v_{1} v_{2} \ldots v_{t} \in E\left(H^{\prime}\right)$ if and only if $v_{1} v_{2} \ldots v_{t}$ is a $K_{t}^{k}$ in $H$. Furthermore set $m=t(t-1)$ and call a balanced $m$-set $A$ an absorbing $m$-set for a balanced $t$-set $T$ if $A$ spans a matching of size $t-1$ in $H^{\prime}$ and $A \cup T$ spans a matching of size $t$ in $H^{\prime}$, in other words, $A \cap T=\emptyset$ and both $H^{\prime}[A]$ and $H^{\prime}[A \cup T]$ contain a perfect matching. Denote by $\mathcal{L}(T)$ the set of all absorbing $m$-sets for $T$. Next, we show that for every balanced $t$-set $T$, there are many absorbing $m$-sets for $T$.

Claim A.1. For every balanced $t$-set $T,|\mathcal{L}(T)| \geq \gamma^{m}\left(\begin{array}{c}n \\ t-1\end{array}\right)^{t} / 2^{t}$.

Proof. Let $T=\left\{v_{1}, \ldots, v_{t}\right\}$ be fixed with $v_{i} \in V_{i}$ for $i \in[t]$. By Proposition 3.1. it is easy to see that there exist at least $(\gamma n)^{t-1}$ edges in $H^{\prime}$ containing $v_{1}$. Since $n_{0}$ was chosen large enough, there are at most $(t-1) n^{t-2} \leq(\gamma n)^{t-1} / 2$ edges in $H^{\prime}$ which contain $v_{1}$ and $v_{j}$ for some $2 \leq j \leq t$. Fix an edge $v_{1} u_{2} \ldots u_{t}$ in $H^{\prime}$ with $u_{j} \in V_{j} \backslash\left\{v_{j}\right\}$ for 
$2 \leq j \leq t$. Set $U_{1}=\left\{u_{2}, \ldots, u_{t}\right\}$ and $W_{0}=T$. For each $2 \leq j \leq t$, suppose we succeed to choose a $(t-1)$-set $U_{j}$ such that $U_{j}$ is disjoint from $W_{j-1}=U_{j-1} \cup W_{j-2}$ and both $U_{j} \cup\left\{u_{j}\right\}$ and $U_{j} \cup\left\{v_{j}\right\}$ are edges in $H^{\prime}$. Then for a fixed $2 \leq j \leq t$ we call such a choice $U_{j}$ good, motivated by $A=\bigcup_{j \in[t]} U_{j}$ being an absorbing $m$-set for $T$.

Note that in each step $2 \leq j \leq t$ there are precisely $t+(j-1)(t-1)$ vertices in $W_{j-1}$. More specifically, for $i \in[t]$, there are at most $j \leq t$ vertices in $V_{i} \cap W_{j-1}$. Thus, the number of edges in $H^{\prime}$ intersecting $u_{j}$ (or $v_{j}$ respectively) and at least one other vertex in $W_{j}$ is at most $(t-1) j n^{t-2}<t^{2} n^{t-2} \leq(\gamma n)^{t-1} / 2$. For each $2 \leq j \leq t$, by Proposition 3.1 there are at least $(\gamma n)^{t-1}-(\gamma n)^{t-1} / 2=(\gamma n)^{t-1} / 2$ choices for $U_{j}$ and in total we obtain $(\gamma n)^{m} / 2^{t}$ absorbing $m$-sets for $T$ with multiplicity at most $((t-1) !)^{t}$

Now, choose a family $F$ of balanced $m$-sets by selecting each of the $\left(\begin{array}{c}n \\ t-1\end{array}\right)^{t}$ possible balanced $m$-sets independently with probability

$$
p=\gamma^{m} n /\left(t^{3} 2^{t+3}\left(\begin{array}{c}
n \\
t-1
\end{array}\right)^{t}\right) .
$$

Then, by Chernoff's inequality, Lemma 3.4 with probability $1-o(1)$ as $n \rightarrow \infty$, the family $F$ satisfies the following properties:

$$
|F| \leq \gamma^{m} n /\left(t^{3} 2^{t+2}\right)
$$

and

$$
|\mathcal{L}(T) \cap F| \geq \frac{\gamma^{2 m} n}{t^{3} 2^{2 t+4}}
$$

for all balanced $t$-sets $T$. Furthermore, we can bound the expected number of intersecting $m$-sets in $F$ by

$$
\left(\begin{array}{c}
n \\
t-1
\end{array}\right)^{t} \times t(t-1) \times\left(\begin{array}{c}
n \\
t-2
\end{array}\right)\left(\begin{array}{c}
n \\
t-1
\end{array}\right)^{t-1} \times p^{2} \leq \frac{\gamma^{2 m} n}{t^{3} 2^{2 t+6}}
$$

Thus, using Markov's inequality, we derive that with probability at least $1 / 2$

$$
F \text { contains at most } \frac{\gamma^{2 m} n}{t^{3} 2^{2 t+5}} \text { intersecting pairs. }
$$

Hence, with positive probability the family $F$ has all properties stated in (7), (8) and (9). By deleting all the intersecting balanced $m$-sets and non-absorbing $m$-sets in such a family $F$, we get a subfamily $F^{\prime}$ consisting of pairwise disjoint balanced $m$-sets, which satisfies

$$
\left|\mathcal{L}(T) \cap F^{\prime}\right| \geq \frac{\gamma^{2 m} n}{t^{3} 2^{2 t+4}}-\frac{\gamma^{2 m} n}{t^{3} 2^{2 t+5}}=\frac{\gamma^{2 m} n}{t^{3} 2^{2 t+5}}
$$

for all balanced $t$-sets $T$. Let $U=V\left(F^{\prime}\right)$ and so $U$ is balanced. Moreover, $U$ is of size at most $t\left|V\left(F^{\prime}\right)\right| \leq t|V(F)| \leq \gamma^{m} n /\left(t^{2} 2^{t+2}\right)$ by (7). For a balanced set $W \subset V \backslash V(M)$ of size $|W| \leq \frac{\gamma^{2 m} n}{t^{2} 2^{2 t+5}}, W$ can be partition in to at most $\frac{\gamma^{2 m} n}{t^{3} 2^{2 t+5}}$ balanced $t$-set. Each balanced $t$-set can be successively absorbed using a different absorbing $m$-set in $F^{\prime}$, so there exists a perfect matching in $H^{\prime}[U \cup W]$. Hence, there is a perfect $K_{t}^{k}$-matching in $H[U \cup$ $W]$. 